\title{
COVID-19 Infection Manifesting with Lumbar Spondylodiscitis Complicating With Psoas Abscess without Pneumonia
}

\author{
Berrin Erok ${ }^{1}$ Kenan Kıbıcı ${ }^{20}$ \\ ${ }^{1}$ Department of Radiology, University of Health Sciences, Prof Dr \\ Cemil Tascıoglu City Hospital, Istanbul, Turkey \\ 2 Department of Neurosurgery, Altınbas University School of Medicine \\ Bahcelievler Medical Park Hospital, Istanbul, Turkey
}

\begin{abstract}
Address for correspondence Berrin Erok, PhD, Department of Radiology, University of Health Sciences, Prof Dr Cemil Tascioglu City Hospital, Istanbul, Turkey (e-mail: drberrinerok@hotmail.com).
\end{abstract}

Indian J Neurosurg

\begin{abstract}
Keywords

- Covid-19

- Sars-CoV-2

- spondylodiscitis

- psoas abscess

- endotheliitis

Extrapulmonary manifestations of COVID-19 (Coronavirus disease 2019) are increasingly recognized. Secondary spinal infections are dangerous complications reported in a few cases in the literature. However, to our knowledge, there is no reported case of a severe spondylodiscitis (SD) complicated with a large psoas abscess in a COVID-19 patient. We would like to report a 43-year-old male patient living in central Anatolia and dealing with farming who presented to the hospital with a complaint of severe back pain. The patient who was given oral treatment with analgesic, anti-inflammatory, and myorelaxant agents was readmitted with increased complaints. His nasopharyngeal swab was positive for COVID-19 without pneumonia on chest computed tomography (CT). He spent the quarantine and treatment period at home but was admitted to our outpatient clinic with a wheelchair with increased complaints and right leg pain preventing daily activities. The control nasopharyngeal swab was negative for COVID-19 but further increase in Creactive protein (CRP) $(152,8 \mathrm{mg} / \mathrm{L})$ and creatine kinase $(C K)$ level $(549 \mathrm{IU} / \mathrm{L})$ were revealed. Lumbar magnetic resonance imaging (MRI) showed SD in the L3-L4 level along with right-sided prevertebral inflammatory soft tissue and a large right psoas muscle abscess. Pyogenic lumbar SD complicated with the right psoas abscess in the setting of COVID-19 was considered and antibacterial treatment was started following hospitalization. On the same day, percutaneous aspiration from the psoas abscess under CT guidance was performed and revealed no growth in the culture. After 3 weeks, follow-up MRI showed worsening of all the bone, soft tissue and disc findings. Myalgia is a common manifestation in viral infections, which was also demonstrated in COVID-19 patients, with possible increase in muscle enzymes. Secondary spinal infections and its soft-tissue complications should be considered in the management of COVID-19 patients with neuromuscular symptoms, and detailed neurological and neurosurgical evaluation should be performed in order to avoid progression and permanent damage.
\end{abstract}

DOI https://doi.org/ 10.1055/s-0041-1741405. ISSN 2277-954X.

\footnotetext{
(c) 2021. Neurological Surgeons' Society of India. All rights reserved. This is an open access article published by Thieme under the terms of the Creative Commons Attribution-NonDerivative-NonCommercial-License, permitting copying and reproduction so long as the original work is given appropriate credit. Contents may not be used for commercial purposes, or adapted, remixed, transformed or built upon. (https://creativecommons.org/ licenses/by-nc-nd/4.0/)

Thieme Medical and Scientific Publishers Pvt. Ltd., A-12, 2nd Floor, Sector 2, Noida-201301 UP, India
} 


\section{Introduction}

COVID-19 (Coronavirus disease 2019) emerged as a primarily respiratory tract infection, however extrapulmonary manifestations are increasingly recognized. Secondary spinal infections are dangerous complications reported in a few cases in the literature. There are few studies regarding the association of COVID-19 with spinal infections. In an investigation, an unusual higher incidence of primary spinal epidural abscess (SEA) following the COVID-19 pandemic was recognized. This study also revealed that the clinical severity of COVID-19 was not correlated to the occurrence of SEA, as in our case. ${ }^{1}$ A case of a COVID-19 patient with lethal spondylodiscitis (SD) associated with thoracic SEA in a 71 year old man without detection of COVID-19 in the abscess cavity was also reported. ${ }^{2}$ However, to our knowledge, there is no reported case of a severe SD complicated with a large psoas abscess in a COVID19 patient.

\section{Case Presentation}

A 43-year-old male with long-term smoking history patient living in central Anatolia and dealing with farming presented to the hospital with a complaint of severe back pain. He was given oral treatment with analgesic, antiinflammatory, and myorelaxant agents but was readmitted to the hospital after a few days when his complaints increased. The initial laboratory results revealed an elevated C-reactive protein (CRP). White blood cell (WBC) count was in the normal range with obviously decreased lymphocyte and mildly increased neutrophil counts. The nasopharyngeal swab was positive for COVID-19 without pneumonia on chest computed tomography (CT). He was sent home and spent the quarantine and treatment period at home but was admitted to our outpatient clinic with a wheelchair with increased complaints and right leg pain preventing daily activities. He was a nonsmoker and there was no previous history of surgical intervention or trauma. On examination, he could only lie in the left lateral decubitus position with extremely painful, limited waist movements. Right lower extremity patellar reflex was hypoactive. Hypoesthesia and severe lumbar paravertebral spasm were present in the L3, L4, and L5 dermatomes. The nasopharyngeal swab was negative for COVID-19 but further increase in CRP $(152.8 \mathrm{mg} / \mathrm{L})$ and creatine kinase (CK) level (549 IU/L) were revealed. The procalcitonin level which is considered as a specific biomarker for bacterial infections was also raised $(0.14 \mathrm{ng} / \mathrm{mL})$. Lumbar magnetic resonance imaging (MRI) showed abnormal signal intensity involving L3-L4 vertebral bodies, the associated intervertebral disc and the end plates along with rightsided prevertebral inflammatory soft tissue which were all hypointense on T1-weighted and hyperintense on short tau inversion recovery (STIR)/T2-weighted images and showed enhancement following contrast administration (-Fig. 1). In addition, an hyperintense encapsulated collection in the right psoas muscle with enhancing thick wall surrounding the central necrotic cavity was revealed (-Fig. 2). Pyogenic
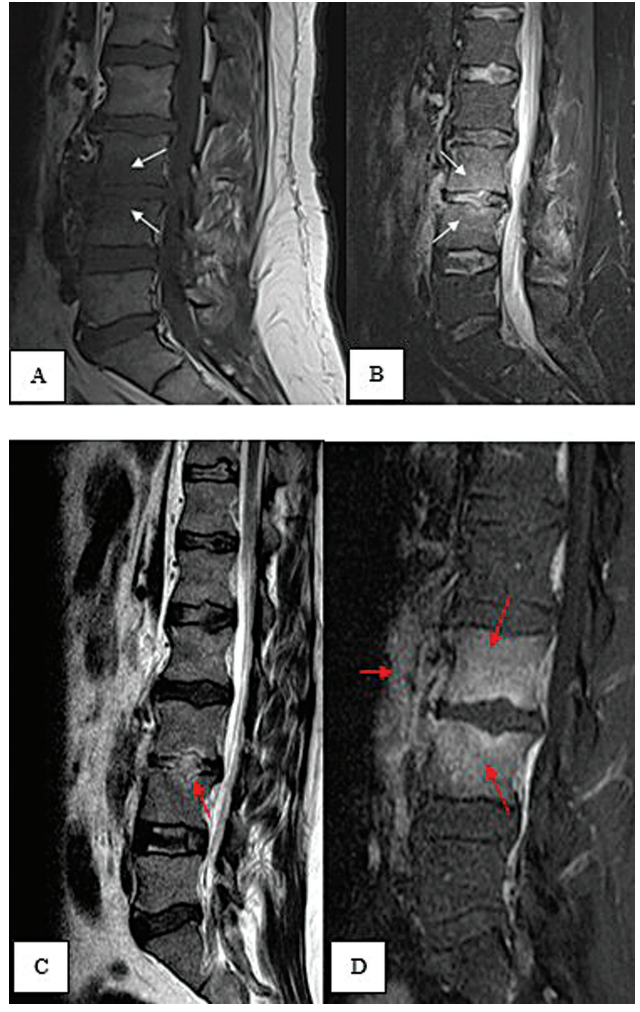

Fig. 1 Lumbar magnetic resonance imaging demonstrating abnormal signal intensity involving L3-L4 vertebral bodies, which is hypointense on T1W (A, white arrows) and hyperintense on short tau inversion recovery ( $\mathrm{B}$, white arrows) images. Abnormally high-signal intensity in the region of end plates and the intervertebral disc is shown on the T2W image (C, red arrow). Note the contrast enhancement in both the involved vertebral bodies and also in the inflammatory prevertebral soft tissue ( $D$, red arrows).

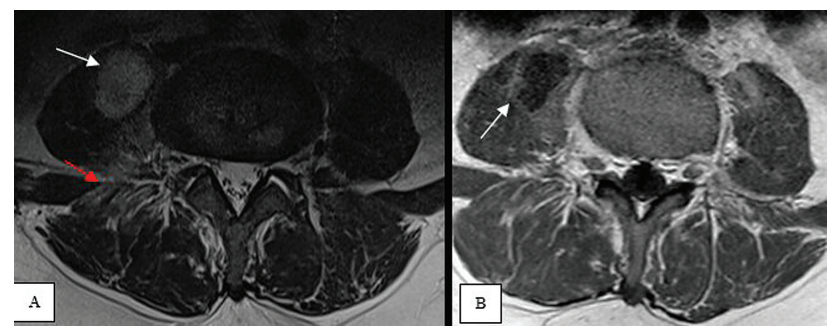

Fig. 2 Lumbar magnetic resonance imaging showing abnormal signal intensity involving the right prevertebral soft tissue on $\mathrm{T} 2 \mathrm{~W}$ axial image ( $A$, red arrow). The hyperintense encapsulated collection in the right psoas muscle ( $A$, white arrow) on axial T2W image and enhancing wall with central necrotic cavity ( $B$, white arrow) on axial enhanced T1W image are demonstrated.

lumbar SD complicated with the right psoas abscess in the setting of COVID-19 was considered and the initial antibacterial treatment was started following hospitalization. On the same day, percutaneous aspiration from the psoas abscess under CT guidance was performed and revealed no growth in the culture. After 3 weeks, follow-up MRI showed worsening of all the bone, soft tissue and disc findings (-Fig. 3 ). 


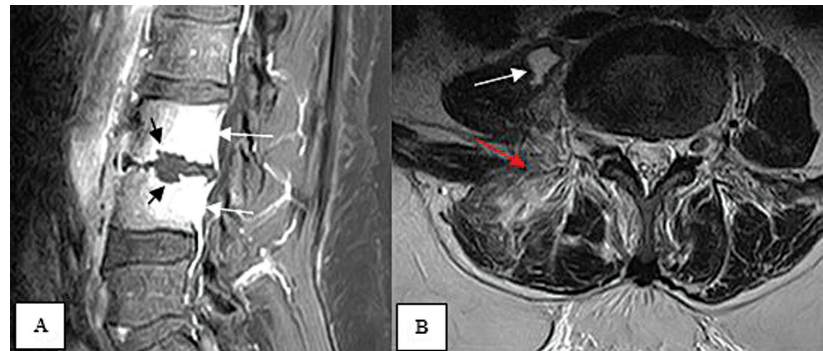

Fig. 3 Follow-up magnetic resonance imaging after 3 weeks showing worsening of the bone (A, white arrows), soft tissue (B, red arrow) and disc findings ( $A$, black arrows). The reduction of the size of the psoas abscess after percutaneous aspiration was also shown (B, white arrow).

\section{Discussion}

Myalgia is a common manifestation in viral infections, which was also demonstrated in COVID-19 patients with possible increase in muscle enzymes, even to the extent of rhabdomyolysis in some cases. ${ }^{3}$ Since the main clinical manifestations of SD are back pain with occasional fever, it can be easily missed in a patient with COVID-19, who is already suffering from these findings in the course of the infection. Neurological examination is important to suspect spinal involvement in these patients. In our patient, the laboratory findings and the MRI features, including lumbar location, the accompanying well-defined but abnormal intervertebral disc signal, involvement of both ventral and lateral parts of the vertebral body without the involvement of facet joints, the large paraspinal soft-tissue abnormality, and the psoas abscess with thick and irregular wall suggested pyogenic SD complicated with psoas abcess. ${ }^{4}$ Tuberculosis (TBC) spondylitis was excluded on account of the absence of previous history of TBC and typical pulmonary changes on chest CT. Due to dealing with farming, brucella SD was also considered in the differential diagnosis and excluded by the negative agglutination test. In addition, the other possible causes of psoas abscess originating from gastrointestinal diseases like appendicitis, diverticulitis or perforation and urinary diseases were also excluded by the abdominopelvic imaging. There was no pervious history of spinal instrumentation or trauma. In COVID-19, the state of immunosuppression and endotheliitis, which have been suggested as underlying causes for worse prognosis in patients with smoking, diabetes, hypertension, obesity and cardiovascular diseases, are the factors predisposing to bacteremia from asymptomatic bacterial colonizations. ${ }^{5}$ In our patient, we also think that immunosuppression and endotheliitis, causing hematogenous dissemination from asymptomatic colonization sites at the state of immunosuppression, which was also facilitated by the long-term smoking history with already damaged endothelium, seem to be the underlying causes for the development of pyogenic complications. A prospective observational study must be done on a large number of cases, so that the pathophysiological aspects can be shown certainly.

\section{Conclusion}

Secondary spinal infections and its soft-tissue complications should be considered in the management of COVID-19 patients with neuromuscular symptoms, and detailed neurological and neurosurgical evaluation is important to suspect from spinal involvement.

\section{Conflict of Interest}

None declared.

\section{References}

1 Talamonti G, Colistra D, Crisà F, Cenzato M, Giorgi P, D’Aliberti G. Spinal epidural abscess in Covid-19 patients. 2020 (e-pub ahead of print). Doi: 10.1007/s00415-020-10211-z

2 Naderi S, Ostadrahimi N, Sheikhrezaei AR, Hänggi D, Muhammad S. COVID-19 infection leading to lethal spondylodiscitis with spinal abscess. Iran J Neurosurg 2020;6(03):151-154

3 Meegada S, Muppidi V, Wilkinson DC III, Siddamreddy S, Katta SK. Coronavirus disease 2019-induced rhabdomyolysis. Cureus 2020; 12(08):e10123

4 Li T, Liu T, Jiang Z, Cui X, Sun J. Diagnosing pyogenic, brucella and tuberculous spondylitis using histopathology and MRI: A retrospective study. Exp Ther Med 2016;12(04):2069-2077

5 Varga Z, Flammer AJ, Steiger P, et al. Endothelial cell infection and endotheliitis in COVID-19. Lancet 2020;395(10234):1417-1418 